University of Nebraska - Lincoln

DigitalCommons@University of Nebraska - Lincoln

$5-1-2000$

\title{
ESR investigations on Ca perovskite
}

M. Chipara

University of Nebraska-Lincoln, mchipara@utpa.edu

Sy-Hwang Liou

University of Nebraska-Lincoln, sliou@unl.edu

C.N. Borca

University of Nebraska-Lincoln

R. Shoemaker

University of Nebraska-Lincoln

Shireen Adenwalla

University of Nebraska-Lincoln, sadenwalla1@unl.edu

See next page for additional authors

Follow this and additional works at: https://digitalcommons.unl.edu/physicsdowben

Part of the Physics Commons

Chipara, M.; Liou, Sy-Hwang; Borca, C.N.; Shoemaker, R.; Adenwalla, Shireen; and Dowben, Peter A., "ESR investigations on Ca perovskite" (2000). Peter Dowben Publications. 165.

https://digitalcommons.unl.edu/physicsdowben/165

This Article is brought to you for free and open access by the Research Papers in Physics and Astronomy at DigitalCommons@University of Nebraska - Lincoln. It has been accepted for inclusion in Peter Dowben Publications by an authorized administrator of DigitalCommons@University of Nebraska - Lincoln. 


\section{Authors}

M. Chipara, Sy-Hwang Liou, C.N. Borca, R. Shoemaker, Shireen Adenwalla, and Peter A. Dowben 


\title{
ESR investigations on Ca perovskite
}

\author{
M. Chipara, Sy-Hwang Liou, C. N. Borca, R. Shoemaker, S. Adenwalla, and P. A. Dowben \\ Department of Physics and Astronomy and the Center for Materials Research and Analysis, \\ Behlen Laboratory of Physics, University of Nebraska, Lincoln, NE 68588-0111, USA.
}

\begin{abstract}
Electron spin resonance studies on fine powders of $\mathrm{La}_{0.65} \mathrm{Ca}_{0.35} \mathrm{MnO}_{3}$, performed in the $\mathrm{X}$ band, are reported. The coexistence of paramagnetic and ferromagnetic phases, in a narrow temperature range close to the Curie temperature, is observed. The electron spin resonance measurements do not support the presence of bipolarons above the Curie temperature.

Temperature dependence of the ESR linewidth is governed by the hopping of polarons and the corresponding activation energy is about $150 \mathrm{meV}$ above $\mathrm{T}_{\mathrm{C}}$.
\end{abstract}

\section{INTRODUCTION}

The $\mathrm{La}_{1-\mathrm{x}} \mathrm{Ca}_{\mathrm{x}} \mathrm{MnO}_{3}$ perovskites are a member of the "colossal magnetoresistance" (CMR) perovskite materials for a doping regime in the range $0.2 \leq \mathrm{x} \leq 0.5$. In the double exchange model [1] the hopping of electrons between $\mathrm{Mn}^{3+}$ and $\mathrm{Mn}^{4+}$ ions (across the $\mathrm{O}$ atom) provides the necessary exchange to impose parallel moments for the two Mn ions. The electrical conduction is largely due to the hopping of the electrons in the $\mathrm{e}_{\mathrm{g}}$ state from $\mathrm{Mn}^{3+}$ to $\mathrm{Mn}^{4+}$ (this band is empty for $\mathrm{Mn}^{4+}$ ions). The model connects the appearance of the magnetic order to the metallic transport. Strong dynamic Jahn-Teller distortions, observed by neutron diffraction on $\mathrm{La}_{0.65} \mathrm{Ca}_{0.35} \mathrm{MnO}_{3}$ provides evidence of changing electron-phonon coupling across the coupled metallic-magnetic transitions, at $240 \mathrm{~K}$ [2-4]. Surface studies confirm strong Jahn-Teller distortion around the Curie temperature in $\mathrm{La}_{0.65} \mathrm{Ca}_{0.35} \mathrm{MnO}_{3}$ suggesting that these distortions are consequences and not a cause $[3,4]$.

\section{EXPERIMENTAL DETAILS}

Electron spin resonance investigations on powders are reported. The sample of $\mathrm{La}_{0.65} \mathrm{Ca}_{0.35} \mathrm{MnO}_{3}$ was prepared as reported elsewhere [2-4]. In the range of temperature investigated the coercive field is smaller than the resonance field by at least one order of magnitude. The resonance spectra were recorded using a Bruker D 200 spectrometer, operating in the $\mathrm{X}$ band $(9 \mathrm{GHz})$. The temperature dependence of electron spin resonance spectra, in the temperature range $200 \mathrm{~K}$ to $350 \mathrm{~K}$ has been studied. 


\section{DISCUSSION}

In the magnetically ordered phase, the electron spin resonance (ferromagnetic resonance mode) consists of a broad asymmetric spectrum (see Figure 1) located at low magnetic fields $(\mathrm{g}>2.00)$. The $\mathrm{g}$ factor, that is proportional to the resonance field, characterize the evolution of a magnetic moment carrying a spin $S$ in an external magnetic field. It is closed to 2.000 for an uncoupled free electron spin and it is sensitive to spin orbit coupling and local magnetic fields [11]. The high value of the $g$ factor in the ferromagnetic phase [11] reflects the presence of such local magnetic field. The resonance spectrum is independent of the orientation of the sample relative to the external magnetic field.

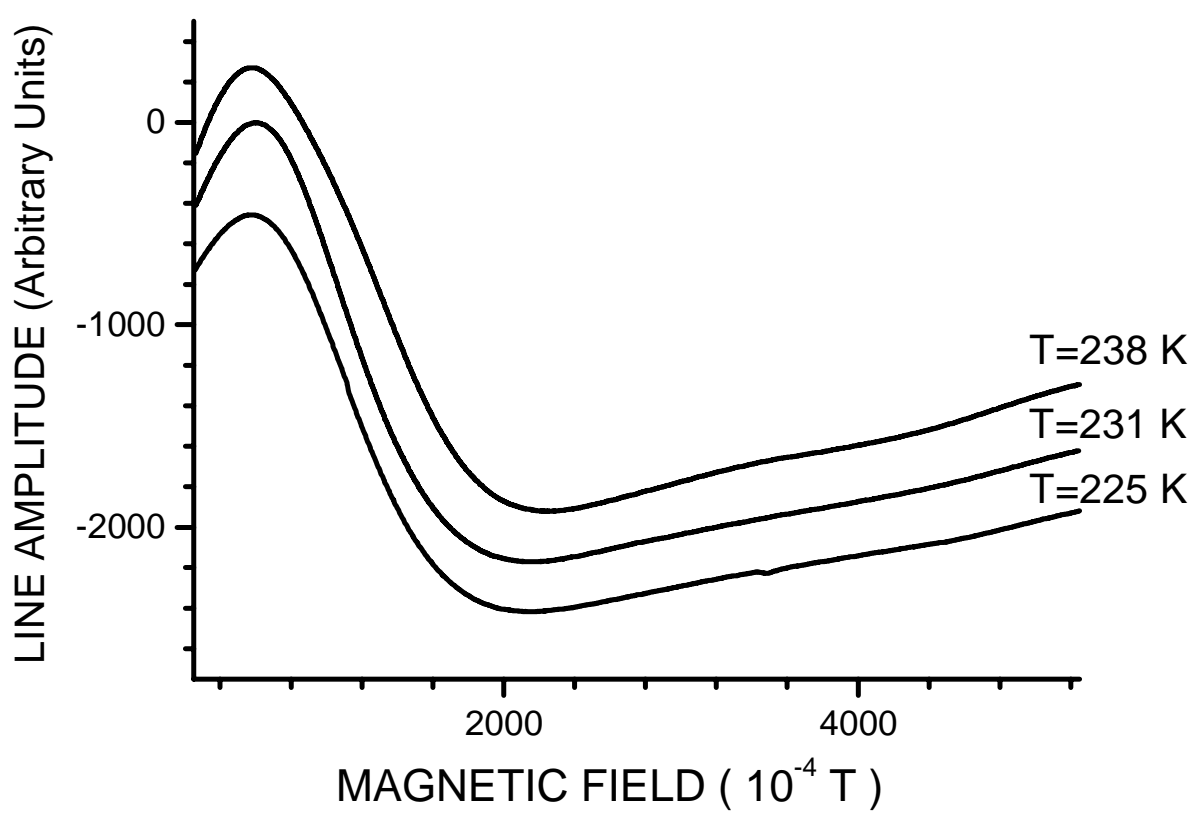

Figure 1. The resonance spectra of $\mathrm{La}_{0.65} \mathrm{Ca}_{0.35} \mathrm{MnO}_{3}$, below $\mathrm{T}_{\mathrm{C}}$.

The presence of domains could split the resonance line, below the Curie temperature, into several components [7]. The asymmetry of the resonance line is due to the competition of two contributions. If the size of the particle is smaller or equal to the skin depth of the microwave field, then this leads to field damping within the sample causing a particular asymmetric line, known as a Dysonian line. As the estimated skin depth in perovskites for $\mathrm{X}$ band measurements is of the order of $0.1 \mathrm{~mm}$ [8-9], the contribution of this process to the resonance line asymmetry is small. The mixing of absorption and dispersion, frequently noticed in the magnetic ordered phase [7], represents the second contribution to line shape anisotropy. This is dominant in our case. The deviation of the resonance line position from the $\mathrm{g}=2.00$ value is due to the local magnetic field and is proportional to the magnetization at saturation.

As the temperature of the sample is raised towards the Curie temperature (Figure 2), the position of the resonance line is shifted towards $\mathrm{H}_{0}=3500 \mathrm{G}$ (corresponding to $\mathrm{g}=2.00$ ). This contribution reflects the decrease of the magnetization at saturation as the temperature approaches (increases towards) the Curie temperature. In the literature, the data concerning the 
temperature dependence of the resonance field are contradictory. Some authors claim a resonance line close to $\mathrm{g}=2.00$ and almost independent of temperature [10]. Recent contributions $[6,8]$ reflect a decrease of the magnetic resonance field as the temperature is decreased below the Curie temperature. The connection between the resonance linewidth, magnetization at saturation, and porosity has been investigated [6]. In a broad temperature range, close to the Curie temperature, two resonance lines, one assigned to the magnetic phase and the other to the paramagnetic phase are noticed (see Figure 2). The coexistence of ferromagnetic and paramagnetic domains, in a temperature range close to Curie temperature was discussed by Borges and collaborators [11].

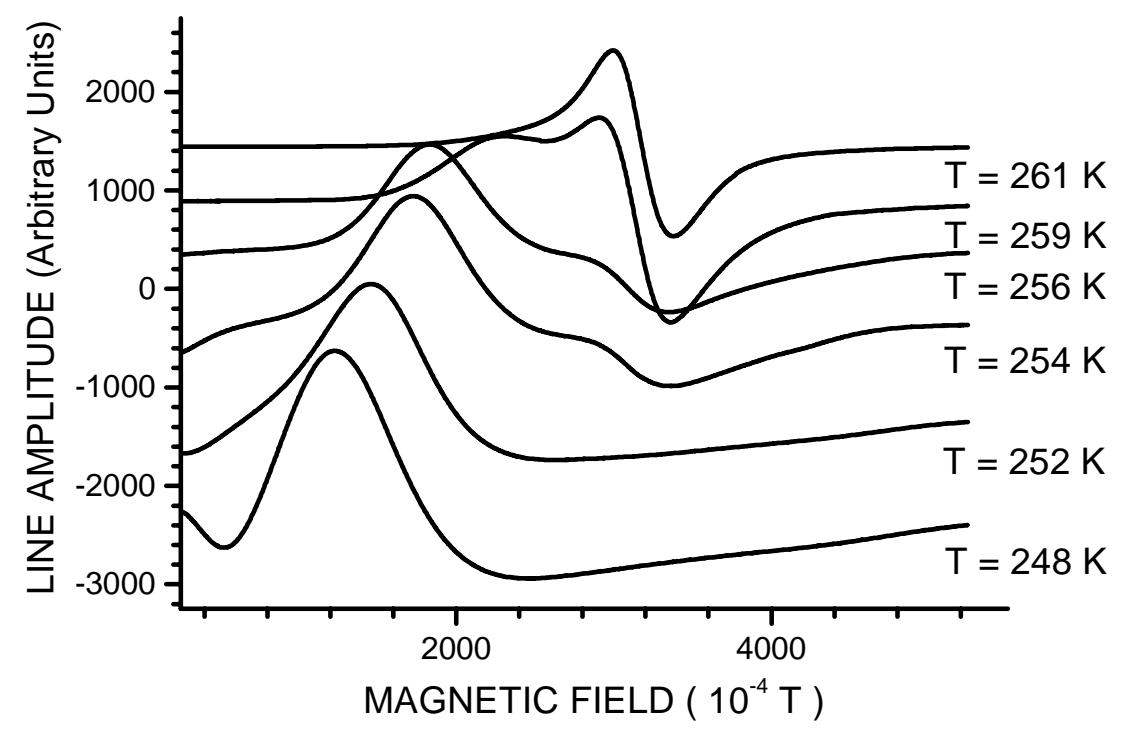

Figure 2. The resonance spectra of $\mathrm{La}_{0.65} \mathrm{Ca}_{0.35} \mathrm{MnO}_{3}$ at the transition from the ferromagnetic to the paramagnetic phase.

The resonance spectra in the paramagnetic phase (see for example in Figure 2 the spectrum recorded at $\mathrm{T}=261 \mathrm{~K}$ ) consist of a narrow line, slightly asymmetric, localized near the $\mathrm{g}=2.00$ value, ascribed to a cluster including both $\mathrm{Mn}^{+}$and $\mathrm{Mn}^{+}$ions [7-10]. The absence of the hyperfine structure, due to the interaction between the uncoupled electronic spin and the nuclear ones is explained by motional effects and/or exchange interactions. The resonance line position (broadest component) increases steadily towards the $\mathrm{g}=2.00$ value (corresponding to the uncoupled electronic spins) as the temperature is increased (see Figure 3) towards the Curie temperature. The shift is caused by the decrease of the local field that is proportional to the magnetization at saturation.

The electrical and magnetic properties of perovskites are related to electric and magnetic polarons and bipolarons. In order to test these hypotheses by electron spin resonance it is important to note that a bipolaron with an effective spin $\mathrm{S}=1$ is expected to provide a resonance line at $\mathrm{g}=4.00$ (i.e. $\mathrm{H}_{0} \approx 1750 \mathrm{G}$ ). We have failed to notice any resonance line at $\mathrm{g}=4.00$ (in the paramagnetic phase). The state specified by $S=1$ is expected to follow a complex dependence of the spin susceptibility versus temperature. The $S=0$ bipolaron should provide no electron spin resonance absorption, in contradiction with the experimental data. This is not the case. Only high above the Curie temperature it is not possible to record the resonance line. The increase of the 
resonance linewidth at high temperatures may be explained by the temperature dependence of relaxation processes above the Curie temperature.

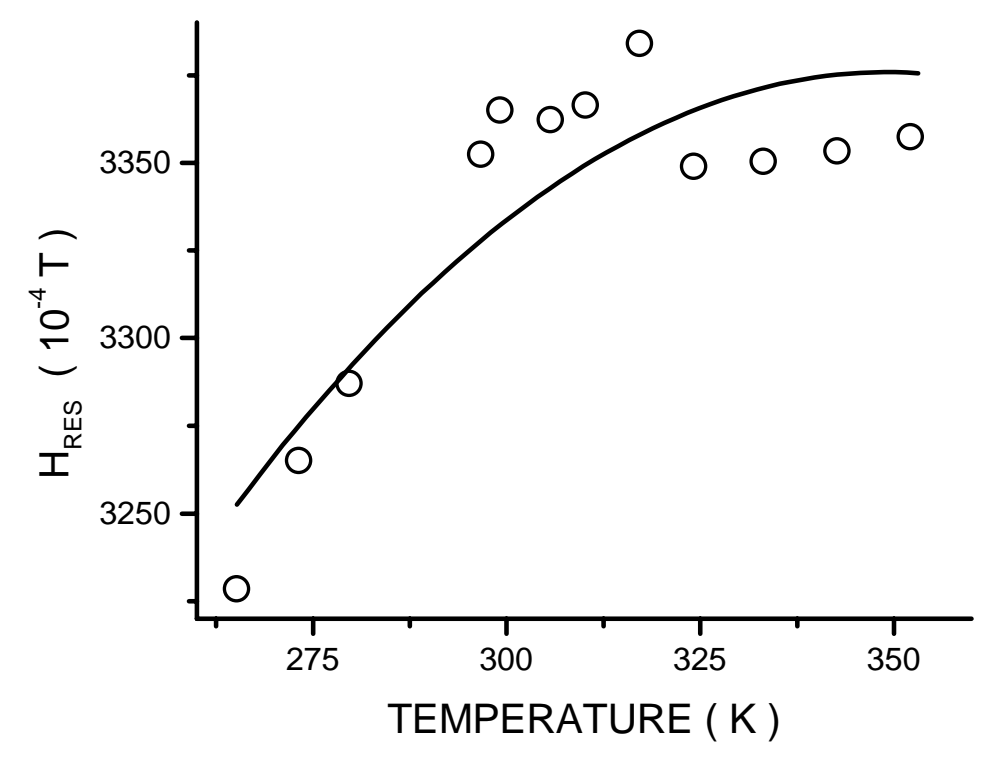

Figure 3. The temperature dependence of the resonance line position.

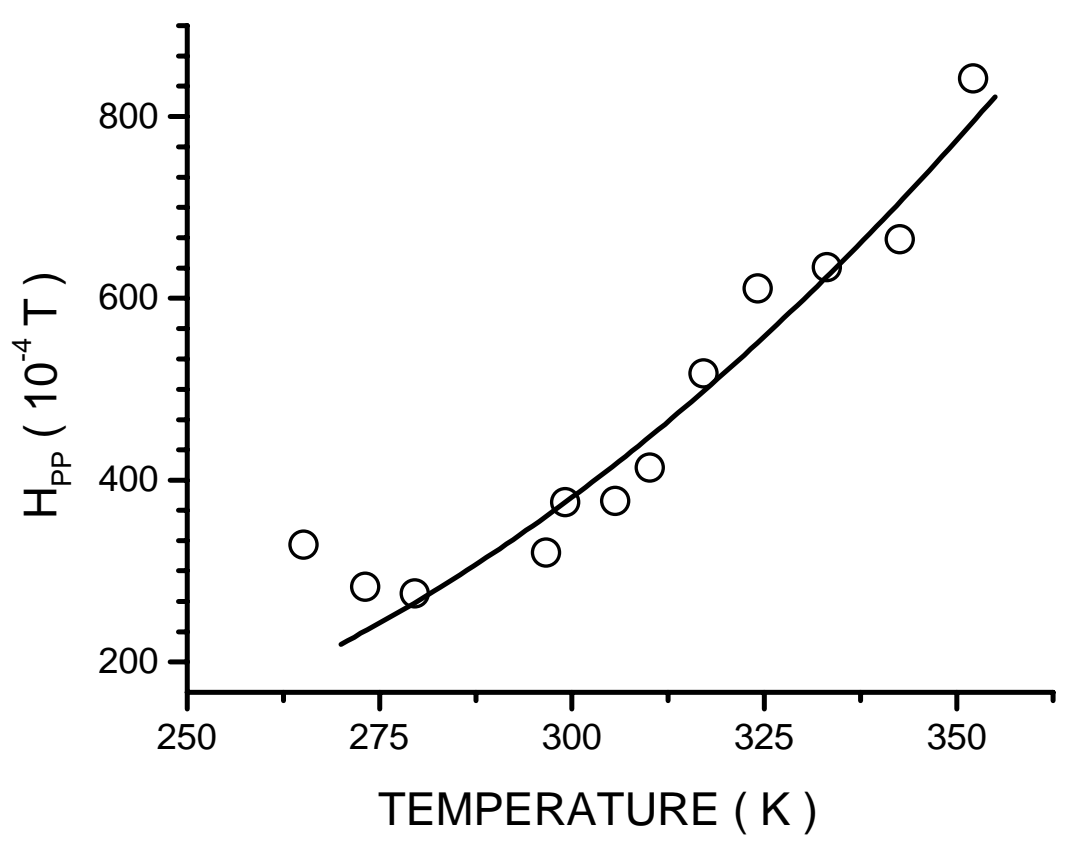

Figure 4. The temperature dependence of the resonance linewidth, above $\mathrm{T}_{\mathrm{C}}$.

As seen in Figure 4, the temperature dependence of the ESR resonance linewidth provides a minimum in $\mathrm{H}_{\mathrm{PP}}$, located at $270 \mathrm{~K}$. The minimum in the temperature dependence of the resonance linewidth occurs at a temperature $\mathrm{T}=1.1 \mathrm{~T}_{\mathrm{C}}[7-11]$, and this is close to a Curie 
temperature of about $250 \mathrm{~K}$. The resonance linewidth, in the magnetically ordered phase decreases as the temperature of the sample is raised towards the Curie temperature. It was noted [9] that the resonance linewidth is proportional to the magnetization at saturation. This qualitative trend is supported by our data. Above the Curie temperature, the resonance linewidth increases as the temperature of the sample is increased. Such a behavior is rather well described by an Arrhenius-like dependence and supports the presence of polarons (see Figure 4):

$$
H_{P P}=H_{P P}^{(0)} \exp -\left(\frac{E_{A}}{K_{B} T}\right)
$$

Where $\mathrm{H}_{\mathrm{PP}}$ is the resonance linewidth, $\mathrm{E}_{\mathrm{A}}$ is the activation energy for the electronic hopping and $\mathrm{K}_{\mathrm{B}}$ represents the Boltzmann constant. The best fit (represented in Figure 4) corresponds to $\mathrm{E}_{\mathrm{A}}=12.4 \mathrm{MJ} / \mathrm{kmole}$ ( about $150 \mathrm{meV}$ ), in good agreement with transport measurements [2].

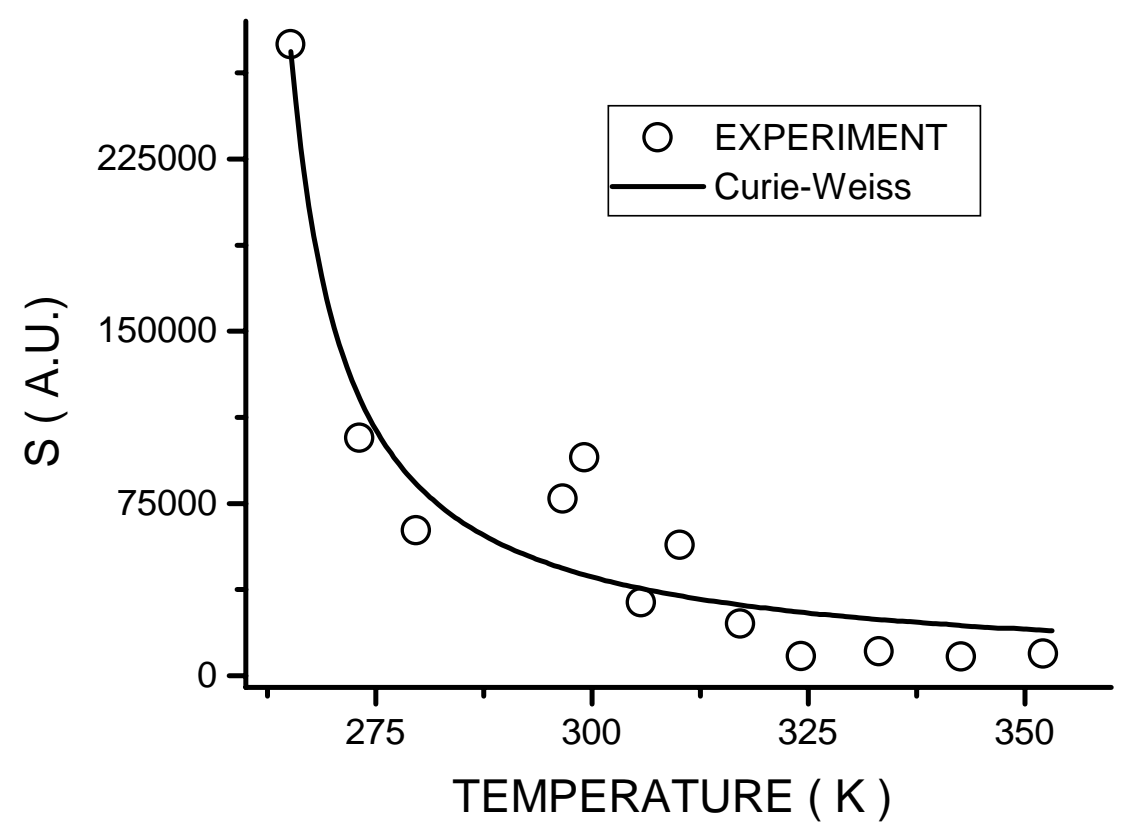

Figure 5. The temperature dependence of the double integral of the resonance spectrum, in the paramagnetic phase.

The temperature dependence of the double integral of the ESR spectrum, $\mathrm{S}$, in the paramagnetic phase, is proportional to the susceptibility of the sample [11]. We have tested an Arrhenius-like behavior and a Curie-Weiss dependence. From Figure 5 it is readily noticed that the Curie-Weiss dependence represents a suitable model for the temperature dependence of S. The Curie temperature, as estimated from EPR data (above the Curie temperature), $\mathrm{T}_{\mathrm{C}}=258 \mathrm{~K}$, is in excellent agreement with the value measured by magnetic methods $[3,4]$ on the same material $\left(\mathrm{T}_{\mathrm{C}}=260 \mathrm{~K}\right)$. 


\section{CONCLUSIONS}

We report on electron spin resonance investigations of $\mathrm{La}_{0.65} \mathrm{Ca}_{0.35} \mathrm{MnO}_{3}$. The coexistence of both paramagnetic and ferromagnetic domains, close to the Curie temperature, was observed. The electron spin resonance spectra are at variance with the presence of bipolarons in this material. The experimental data are consistent with a polaronic hopping (in the paramagnetic phase). The double integral of the resonance line obeys Curie-Weiss dependence with a Curie temperature of about $258 \mathrm{~K}$. This value is in good agreement with the Curie temperature

estimated from the temperature dependence of the resonance linewidth and with magnetic data.

\section{ACKNOWLEDGEMENTS}

This work was supported by NSF through grants \#DMR-98-06308, DMR 98-02126 and by the Center for Materials Research and Analysis (CMRA) at the University of Nebraska.

\section{REFERENCES}

1. P.W. Anderson and H. Hasagawa, Phys. Rev., 100, 675 (1955) .

2. J. M. D. Coey and M. Virett, Advances in Phys., 48, 2, 167 (1999).

3. P. Dai, J. Zhang, H. A. Mook, S. H. Liou, P. A. Dowben, and E. W. Plummer, Phys. Rev. B, 54, R3694 (1996).

4. J. D. Zhang, D. N. McIlroy, P. A. Dowben, S. H. Liou, R. F. Sabirianov, and S. S. Jaswal, Sol. State Commun., 97, 1, 39 (1996).

5. Ch. Kittel, Introduction to Solid State Physics, 7th ed. (John Wiley \& Sons, New York, 1996) pp. 503.

6. F. Rivadulla, L. E. Hueso, C. Jardon, C. Vazquez-Vazquez, M. A. Lopez-Quintela, J. Rivas, M. T. Causa, C. D. Ramos, and R. D. Sanchez, J. Mag. Mag. Mat., 196-197, 470 (1999).

7. U. Ebbels, P. E. Wigen, and K. Ounadjela, J. Magn. Magn. Mat., 177-181, 1239 (1998).

8. V. A. Ivanshin, J. Deisenhofer, H.-A. Krug Von Nidda, A. Loidl, A. A. Mukhin, A. M. Balbashov, and M. V. Eremin, Phys. Rev. B, 61, 9, 6213 (2000).

9. C. Rettori, D. Rao, J. Singley, D. Kidwell, S. B. Oseroff, M. T. Causa, J. J. Neumeier, K. J. McClellan, S-W. Cheong, and S. Schultz, Phys. Rev. B, 55, 5, 3083 (1997).

10. J. S. Ramachandran, S.M. Bhagat, and J.L. Peng, Sol. State Commun., 96, 3, 127 (1995).

11. R. P. Borges, F. Ott, R. M. Thomas, V. Skumryev, J. M. D. Coey, J. I. Arnaudas, and L. Rann, Phys. Rev. B, 60, 18, 12847 (1999). 